\title{
Apparent absence of nicotine-induced conditioned place preference in rats
}

\author{
P.B.S. Clarke and H.C. Fibiger
}

Division of Neurological Sciences, Department of Psychiatry, University of British Columbia 2255 Wesbrook Mall, Vancouver, B.C., Canada V6T 2A1

\begin{abstract}
The conditioned place preference (CPP) paradigm was used in order to assess the reinforcing actions of nicotine in rats. Subjects were tested in "unbiased" two-compartment shuttle boxes, so-called because neither compartment was consistently preferred prior to drug conditioning. In the first experiment, subjects that were initially drug naive showed neither a preference nor an aversion to the compartment that had been paired on four occasions with injection of nicotine $(0.2-0.8 \mathrm{mg} / \mathrm{kg} \mathrm{SC})$; a similar result occurred in another group given daily injections of nicotine in the home cage prior to the experiment. In a second experiment, nicotine $(0.4,0.8 \mathrm{mg} / \mathrm{kg} \mathrm{SC})$ again failed to produce a CPP, whereas marked CPPs were seen in parallel groups of rats tested with either $d$-amphetamine or methylphenidate. Although nicotine has been reported to produce conditioned place preference, the present results suggest that it is not a robust phenomenon.
\end{abstract}

Key words: Conditioned place preference - Reinforcement - Nicotine - $d$-Amphetamine - Methylphenidate - Rats

Nicotine is voluntarily self-administered by man (Henningfield and Goldberg 1983) and by laboratory animals (Goldberg et al. 1981; Cox et al. 1984), but the neural mechanisms that underlie the drug's positive reinforcing action have not been elucidated. In rats, nicotine self-administration occurs, but in studies to date, rates of responding and of drug infusion have been modest (see Stolerman 1987 for review). The conditioned place preference (CPP) paradigm provides an alternative and convenient method for studying the reinforcing properties of drugs in animals. In this procedure, one compartment of a shuttle box is repeatedly paired with the administration of a drug, and it is usually assumed that the positive reinforcing properties of the drug become associated with distinctive environmental cues present in the drug-paired compartment. Subsequently, the animal is allowed free access to both compartments, and a shift of side preference is taken as an indication of the drug's reinforcing properties. Many drugs which are intravenously self-administered by laboratory animals produce CPPs. Such drugs include opiates (e.g. Kumar 1972), $d$-amphetamine (Sherman et al. 1980), cocaine (Spyraki et al. 1982a) and, recently, nicotine itself (Fudala et al. 1985; Fudala and Iwamoto 1985).

Offprint requests to: P.B.S. Clarke
Many demonstrations of drug-induced place preference, including those with nicotine (Fudala et al. 1985; Fudala and Iwamoto 1985), have employed shuttle boxes in which rats prefer one compartment over the other, and in which the less preferred side is assigned for drug conditioning. This introduces an interpretational problem, since a druginduced shift of preference could reflect an ability of the drug to reduce the aversiveness of the less preferred compartment, for example by suppressing neophobia. In order to circumvent this problem, we employed shuttle boxes in which there was no consistent initial preference for one side over the other. In the first experiment, the effects of nicotine were examined in a dose range which has been reported to produce a dose-related CPP (Fudala and Iwamoto 1985). An additional group of rats received injections of the drug in their home cages, on several days prior to the experiment, since the acute actions of nicotine are markedly altered by repeated pre-exposure (Clarke and Kumar $1983 \mathrm{a}, \mathrm{b}$ ). In the second experiment, an identical procedure was adopted, and the acute effects of two selected doses of nicotine were re-examined in a new group of drug-naive subjects. Comparisons were also made with $d$-amphetamine and methylphenidate, both of which produce CPPs in this apparatus (Mithani et al. 1986).

\section{Materials and methods}

Subjects. Male hooded rats (Charles River), initially weighing $320-440 \mathrm{~g}$ (experiment 1) or 270-350 g (experiment 2), were housed three or four per cage in a room illuminated from 0800 to $2000 \mathrm{~h}$, with continuous access to food and water. Subjects were adapted to laboratory conditions for at least 2 weeks and were handled on 5 consecutive days prior to each experiment. Of the 50 subjects of experiment 1 , one-fourth had received a single injection of diazepam $(2.5 \mathrm{mg} / \mathrm{kg}, n=6)$ or of the GABA agonist THIP $(5 \mathrm{mg} / \mathrm{kg}, n=7) 2$ weeks before, and they were randomly assigned to drug conditioning groups.

Apparatus. Each animal was tested in one of four shuttle boxes $(80 \times 25 \mathrm{~cm}, 35 \mathrm{~cm}$ high) divided into two compartments of equal floor area $(34 \times 25 \mathrm{~cm})$ and joined by a tunnel $(8 \times 8 \times 6 \mathrm{~cm})$ that could be closed at both ends by guillotine doors. A tray of sawdust was located under the cage floor. In each box, the two compartments differed in the appearance of the walls (uniform brown versus black and white stripes $1 \mathrm{~cm}$ wide) and in the type of floor (parallel 
rods spaced $1.2 \mathrm{~cm}$ apart versus $1.2 \mathrm{~cm}$ wire mesh). The wall and floor cues were counterbalanced so that as far as possible, an equal number of animals in each drug group were assigned for drug-conditioning to each of the four combinations of wall and floor: (1) brown walls and grid floor (2) brown walls and mesh floor (3) striped walls and grid floor and (4) striped walls and mesh floor. Translucent Plexiglas lids allowed for diffuse, low-level illumination from four fluorescent lights in the ceiling. Each shuttle box was mounted on a fulcrum, and its position was detected by microswitches. Solid state equipment was employed to record the time spent on either side and the number of crossings from one compartment to the other.

Drugs. (-)-Nicotine $\mathrm{H}(+)$ bitartrate (BDH), d-amphetamine sulphate (SKF), and methylphenidate $\mathrm{HCl}$ (CIBA) were dissolved in $0.9 \% \mathrm{w} / \mathrm{v} \mathrm{NaCl}$ solution (saline). The nicotine solution was neutralised to $\mathrm{pH} 7.2 \pm 0.1$ with $\mathrm{NaOH}$ solution. Drugs were aliquotted and stored at $-80^{\circ} \mathrm{C}$ and thawed on the day of use. Injections were subcutaneous, made into the flank in a volume of $1 \mathrm{ml} / \mathrm{kg}$. Doses of nicotine refer to the base of the compound, those of $d$-amphetamine and methylphenidate to the salt.

Behavioural procedure. The behavioural procedure was the same in the two experiments. Testing was conducted between 0800 and $1600 \mathrm{~h}$ for 16 consecutive days. There were three phases to each experiment: pretest (phase 1: days 1-5), conditioning (phase 2: days 6-13) and post-test (phase 3: days 14-16). During phases 1 and 3, the same test procedure was employed: rats were individually weighed, then placed in groups of four in a plastic holding cage and left undisturbed for $10 \mathrm{~min}$ in the testing room. Next, each rat was placed in one of the compartments (hereafter termed the start side) of a shuttle box, and between 15 and $45 \mathrm{~s}$ later, the test session was started. Test sessions were of $900 \mathrm{~s}(15 \mathrm{~min})$ duration. Throughout phases 1 and 3 , the guillotine doors remained open, and the time spent in each compartment, as well as the number of crossings made from the start side to the non-start side, was recorded.

Phase 2 comprised 8 days. Groups of four preweighed rats were left in holding cages for $20 \mathrm{~min}$ in the testing room, then injected in rapid succession with saline or drug, depending on drug group and day. Subjects were then briefly returned to the holding cage, so that they were all introduced to the shuttle boxes $2 \mathrm{~min}$ after injection. Conditioning sessions lasted for $30 \mathrm{~min}$. On days $6,8,10$ and 12 (drug days), rats received drug injections and were confined to the non-start ("drug-paired") side of the box. On alternate days (control days), rats received saline injections and were confined to the start ("control-paired") side. As far as possible, testing was counterbalanced so that equal numbers of rats from each drug group were tested in each shuttle box.

Analysis of data. Multivariate and univariate analyses of variance were performed using a software package (SPSSX, Chicago IL), each subject serving as its own control wherever possible. The principal dependent measure was the amount of time spent on the drug-paired (non-start) side of the box, expressed either in absolute terms (for pretests) or as the difference from baseline pretests (for post-tests). In order to avoid positive biasing of the $F$ test, which may occur in designs having repeated measures on the same subjects, the following procedure, recommended by Kirk (1968), was adopted. A repeated measures univariate analysis of variance was performed: a non-significant $F$ value refers to a conventional $F$ test, whilst a significant $F$ value refers to the Geisser-Greenhouse Conservative $F$ test. Where the symmetry conditions permitting univariate tests were not met (see SPSSX User's Guide p 522), or where only the conventional $F$ test gave a significant result, an exact multivariate test (Hotelling's T2) was employed. Comparisons between control (i.e. saline) and other drug groups were made with a multiple range test (Dunnett 1955).

Experiment 1: tests with nicotine. Subjects were randomly assigned to five groups of ten rats each, receiving the following treatments on drug days (Phase 2): Group 1 - saline; Group 2 - nicotine $0.2 \mathrm{mg} / \mathrm{kg}$; Group 3 - nicotine $0.4 \mathrm{mg} /$ $\mathrm{kg}$; Group 4 - nicotine $0.8 \mathrm{mg} / \mathrm{kg}$; Group 5 - nicotine $0.4 \mathrm{mg} / \mathrm{kg}$. In addition, subjects of Group 5 received daily injections of nicotine $(0.4 \mathrm{mg} / \mathrm{kg} \mathrm{SC})$ in their home cages for 7 consecutive days ending 2 days before the start of phase 1.

Experiment 2: Tests with nicotine, d-amphetamine, and meth$y$ lphenidate. Subjects were randomly assigned to five drug groups ( $n=10$ per group) as follows: Group 1 - saline; Group 2 - methylphenidate $5 \mathrm{mg} / \mathrm{kg}$; Group 3 - $d$-amphetamine $1.5 \mathrm{mg} / \mathrm{kg}$; Group 4 - nicotine $0.4 \mathrm{mg} / \mathrm{kg}$; Group 5 - nicotine $0.8 \mathrm{mg} / \mathrm{kg}$. All subjects were drug naive at day 1 .

\section{Results}

\section{Experiment 1: Tests with nicotine}

Across the 5 days of pretests, the amount of time spent on the non-start side varied (main effect of DAYS : $F=4.27$, $d f 4,46, P<0.01$ ). Helmert contrast analysis (SPSSX User's Guide $\mathrm{p} 477$ ) was used in order to test whether the group mean score of a given pretest was significantly different from the mean score of any subsequent pretests. This analysis showed the first two pretests to be different from the last three, which did not differ significantly. Across the final three pretests, the time spent on the non-start side did not differ between the randomly allocated groups (Table 1 ; main effect of GROUP: $F=0.05$, df $4,45, P>0.05$ ), and the interaction between GROUP and DAY was also nonsignificant $(F=1.02$, df 8,90). Therefore, for each subject, a baseline score was calculated as the mean of the individual scores obtained on these three days (Table 1). During this baseline period, there was no significant preference for either compartment, the mean \pm SEM time spent on the nonstart side being $414.7 \pm 24.9 \mathrm{~s}(n=50)$ out of $900 \mathrm{~s}$.

Following the conditioning phase, the time spent on the drug-paired (non-start) side differed across the three post-tests (main effect of DAY: $F=7.38, d f 1,45, P<0.01$; Table 1), but this difference was independent of treatment group (DAY $\times$ GROUP interaction: $F=0.43$, df $8,90, P>$ 0.05 ).

Shifts of side preference were assessed by the difference of time spent on the drug-paired side during the three posttests compared to the pretest baseline. A positive value indicates an increased preference for the drug-paired (non-start) side. For initial analyses, the data from the three post-tests were pooled for each subject. Conditioning with saline on both sides of the box (Group 1) did not result in a signifi- 
Table 1. Time (s) spent on drug-paired side during pretests and change from baseline during post-tests

Group mean $\pm \operatorname{SEM}(n=10$ per group)

\begin{tabular}{|c|c|c|c|c|c|c|c|}
\hline \multirow[t]{2}{*}{ Group } & \multicolumn{4}{|c|}{ Pretests } & \multicolumn{3}{|c|}{ Post-test baseline } \\
\hline & 3 & 4 & 5 & Baseline $^{b}$ & 1 & 2 & 3 \\
\hline
\end{tabular}

Experiment 1

\begin{tabular}{|c|c|c|c|c|c|c|c|}
\hline Saline & $456 \pm 69$ & $408 \pm 73$ & $430 \pm 65$ & $431 \pm 59$ & $73 \pm 51$ & $38 \pm 42$ & $13 \pm 49$ \\
\hline Nic 0.2 & $425 \pm 49$ & $402 \pm 58$ & $402 \pm 60$ & $410 \pm 49$ & $64 \pm 66$ & $8+82$ & $-35 \pm 53$ \\
\hline $\mathrm{Nic} 0.4$ & $390 \pm 55$ & $425 \pm 76$ & $419 \pm 79$ & $412 \pm 67$ & $45 \pm 51$ & $-23+78$ & $-42+69$ \\
\hline Nic 0.8 & $386 \pm 49$ & $489 \pm 69$ & $396 \pm 68$ & $424 \pm 53$ & $58 \pm 68$ & $-49 \pm 25$ & $-27 \pm 50$ \\
\hline $\mathrm{Nic} 0.4^{\mathrm{a}}$ & $420 \pm 63$ & $426 \pm 77$ & $344 \pm 54$ & $397 \pm 61$ & $4 \pm 62$ & $-97 \pm 51$ & $-36 \pm 65$ \\
\hline \multicolumn{8}{|c|}{ Experiment 2} \\
\hline Saline & $460 \pm 37$ & $422 \pm 42$ & $470 \pm 41$ & $451 \pm 34$ & $-40 \pm 62$ & $-56 \pm 58$ & $-11 \pm 54$ \\
\hline MPD 5.0 & $416 \pm 39$ & $381 \pm 44$ & $453 \pm 46$ & $417 \pm 35$ & $97 \pm 33$ & $178+44$ & $139 \pm 47$ \\
\hline AMP 1.5 & $377 \pm 35$ & $414 \pm 35$ & $409 \pm 32$ & $400 \pm 26$ & $232 \pm 39$ & $187 \pm 49$ & $131 \pm 30$ \\
\hline Nic 0.4 & $428 \pm 31$ & $433 \pm 34$ & $419 \pm 26$ & $427 \pm 15$ & $89 \pm 40$ & $33 \pm 32$ & $17 \pm 47$ \\
\hline Nic 0.8 & $442 \pm 40$ & $428 \pm 34$ & $481 \pm 30$ & $450 \pm 27$ & $33 \pm 40$ & $-31 \pm 31$ & $-66 \pm 32$ \\
\hline
\end{tabular}

a This group received daily injections of nicotine $0.4 \mathrm{mg} / \mathrm{kg}$ before the start of the experiment

${ }^{b}$ Baseline scores are the mean of pretests 3,4 and 5

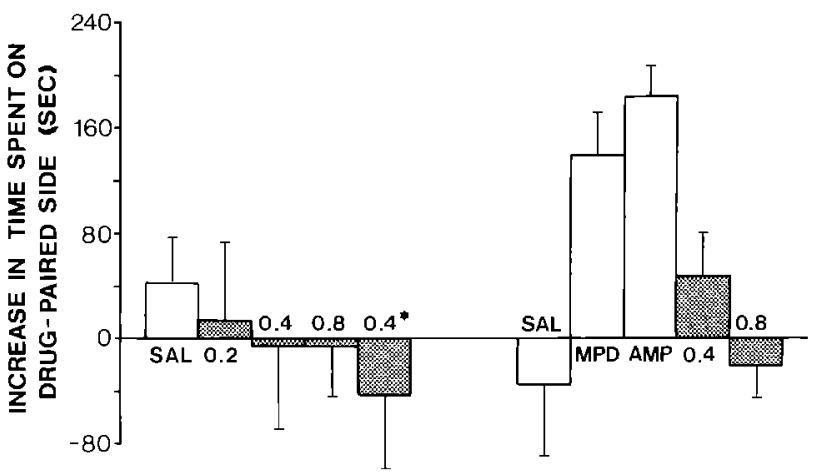

Fig. 1. Shifts of side preference following conditioning with nicotine, $d$-amphetamine or methylphenidate. During the drug conditioning phase, rats were placed in one side of a shuttle box after $\mathrm{SC}$ injection of saline (control group), nicotine (shaded, $\mathrm{mg} / \mathrm{kg}$ dose as shown), $d$-amphetamine ( $A M P-1.5 \mathrm{mg} / \mathrm{kg}$ ), or methylphenidate $(M P D-5 \mathrm{mg} / \mathrm{kg})$; on other days, subjects received saline on the other side. The vertical axis shows the mean increase in time spent on the drug-paired side of the test box during the three post-conditioning tests compared to the pre-conditioning baseline. Thus a positive value indicates an increased preference for the drug-paired side. Data represent mean \pm SEM difference scores $(n=10$ per group). In experiment 1 (left panel), nicotine $(0.2-0.8 \mathrm{mg} / \mathrm{kg}$ ) failed to produce a significant preference shift, even in subjects treated with the drug for 1 week before the experiment (shaded, $0.4^{*}$ ). In experiment 2 (right panel), $d$-amphetamine and methylphenidate produced marked place preferences, but nicotine was again ineffective

cant shift of side preference (Fig. 1). In addition, conditioning with nicotine failed to produce a significant shift of preference (main effect of GROUP: $F=0.36$, $d f 4,45, P>$ 0.05 ; Fig. 1). This analysis did not exclude the possibility that nicotine might have produced a CPP which, through rapid extinction, was only detectable on the first post-test. This possibility was ruled out by subsequent analysis which showed that nicotine did not produce a significant shift of preference between the baseline pretests and the first post-test (main effect of GROUP: $F=0.20$, df $4,45, P>$
0.05; Table 1). Within $2 \mathrm{~min}$ of injection of nicotine, the subjects became ataxic and, at the higher doses, transient prostration occurred. These motor effects, which lasted for a few minutes, appeared to wain across successive drug sessions, and were not visible in rats which had received home cage injections prior to the experiment.

Subjects were moderately active during both pretests and post-tests. The mean + SEM number of crossings into the drug-paired side made per pretest session was $9.3 \pm 0.5$ $(n=50)$, and following the conditioning phase, there was a small increase of $1.34 \pm 0.54$ crossings per session $(t=2.48$, df $49, P<0.01$ ). The baseline number of crossings and changes observed after the conditioning phase did not differ significantly between groups.

\section{Experiment 2: tests with nicotine, $d$-amphetamine and methylphenidate}

As in experiment 1, the time spent in the non-start side of the shuttle boxes differed across the five pretests (main effect of DAY: $F=3.24$, df 4,46, $P<0.02$ ), and Helmert contrast analysis indicated pooling of scores for the final three pretests. Baseline data, calculated in this way, did not differ between treatment groups (main effect of GROUP: $F=0.60, d f 4,45, P>0.05$; GROUP $\times$ DAY interaction: $F=0.58$, df $8,90, P>0.05)$, and there was no significant side preference $($ mean \pm SEM time on non-start side $=$ $428.9 \pm 12.5 \mathrm{~s}-$ see Table 1$)$.

Following conditioning, the time on the drug-paired (non-start) side did not differ between the three post-tests (main effect of DAY : $F=2.18, d f 2,90, P>0.1$ ), irrespective of treatment group (interaction of DAY $\times$ GROUP: $F=$ 1.77, df $8,90, P>0.05$ ). Therefore, changes of side preference were assessed, as in experiment 1 , as the mean increase in time spent on the drug-paired side during the three posttests compared to the mean score of the final three pretests. Conditioning with saline on both sides of the apparatus did not alter side preference (Table 1 and Fig. 1, group 1). Changes of side preference differed between the treatment groups (main effect of GROUP: $F=7.16$, df $4,45, P<$ 
0.001). Dunnett's multiple range test was then employed to compare the change of side preference which occurred in each of the four drug treatment groups with that of the control (saline) group: both methylphenidate and $d$ amphetamine produced significant place preferences $(P<$ 0.01 for each), whereas neither dose of nicotine was effective $(P>0.05$ for each), as shown in Fig. 1. Although the place preference produced by $d$-amphetamine appeared to wain across successive post-tests (See Table 1), this change was not statistically significant (main effect and linear trend of DAY : $P>0.08$ for both).

Further analysis examined whether nicotine had produced a transient CPP, detectable only on the first post-test. Changes of side preference between the final three pretests and the first post-test differed significantly between the five treatment groups (main effect of GROUP: $F=5.11$, $d f 4,45$, $P<0.002$ ). Compared to the saline control group, neither group of rats injected with nicotine showed a significant shift of preference between the baseline pretests and the first post-test (Dunnett's test: $P>0.1$ for both). Methylphenidate produced a marginally non-significant shift ( $P=$ $0.05)$, whereas the effect of amphetamine was clear $(P<$ 0.01 ).

\section{Discussion}

In both experiments described above, nicotine failed to produce a conditioned place preference. Pronounced CPPs were, however, seen in parallel groups of rats conditioned with either methylphenidate or $d$-amphetamine, thus confirming previous findings from this laboratory (Mithani et al. 1986). In one respect, the present results differ from those of Mithani et al.; extinction to the stimulant-induced CPPs was not observed over successive post-tests. The source of this discrepancy is not clear.

Although nicotine did not produce a CPP, the drug was clearly behaviourally active, demonstrated by the occurrence of ataxia and prostration shortly after injection, as previously reported (Clarke and Kumar 1983a, b). In contrast, Fudala and colleagues have reported dose-related CPPs following conditioning with nicotine in the same dose range as employed in the present study (Fudala et al. 1985; Fudala and Iwamoto 1985). In their studies, nicotine produced a shift towards the less preferred compartment with which it had previously been paired. This shift did not apparently result in an absolute preference for the drug-associated side. Thus it is possible that nicotine produced the shift of preference by reducing neophobia associated with the non-preferred side, particularly since subjects were not exposed to the apparatus before the conditioning phase, rather than by providing a positive reinforcing action to which environmental cues could be conditioned.

The experimental method employed in the present study differed in a number of respects from that of Fudala and colleagues. These investigators employed testing boxes in which subjects consistently preferred one compartment and received conditioning drug trials in the less preferred side; no tests of baseline preference were performed prior to conditioning with nicotine; three compartments were employed, of which the two compartments used for conditioning differed in colour and in shape, and were further distinguished by olfactory cues; three rather than four drug and saline conditioning trials were given; subjects were placed in the apparatus with no delay following injection; albino rats were used. It is unclear which if any of these factors is responsible for the lack of nicotine-induced CPP in the present study.

(1) The use of five pretests served to establish baseline performance and may have reduced fear associated with a novel environment, but may also have resulted in some degree of latent inhibition. If this occurred, it was not sufficiently great to prevent the appearance of CPPs following treatment with methylphenidate or $d$-amphetamine (experiment 2) or with heroin (Bozarth and Wise 1981). Moreover, the presence or absence of five pretests was not found to be a significant factor in the degree of place conditioning obtained with naloxone (Mucha and Iversen 1984).

(2) In the present study, some central actions of nicotine were already apparent in the $2 \mathrm{~min}$ between injection and introduction of the subjects to the apparatus; this was not the case for amphetamine and methylphenidate. In the terminology of Pavlovian conditioning, the failure to find a CPP with nicotine may reflect the initiation of the unconditioned stimulus (nicotinic action) before the conditioned stimulus (apparatus). This explanation cannot be dismissed at present, but seems unlikely, insofar as other investigators have successfully demonstrated CPPs with amphetamine and morphine with even longer delays between injection and introduction to the apparatus (Phillips and LePiane 1980; Sherman et al. 1980; Spyraki et al. 1982 b).

(3) A strain difference is unlikely to account for the lack of nicotine-induced CPP, insofar as hooded and albino rats respond to nicotine in a similar fashion in other behavioural paradigms (Clarke and Kumar 1983b; Clarke 1984; Clarke and Kumar 1984).

Could the lack of nicotine-induced CPP reflect the presence of aversive as well as rewarding effects? Certainly, nicotine exerts potent emetic and dysphoric actions in human non-smokers (Beckett et al. 1971; Henningfield and Goldberg 1983), and emesis is also seen in cats and dogs (Laffan and Borison 1957) and monkeys (Deneau and Inoki 1967). Rats lack an emetic reflex, but nicotine acts as a potent unconditioned stimulus in the conditioned taste aversion (CTA) pradigm (Kumar et al. 1983). Monkeys and human subjects will work to avoid intravenous injections of the drug that would be self-administered under different experimental conditions (Henningfield and Goldberg 1983). Despite these considerations, there is little direct evidence to suggest that nicotine should manifest an aversive component in the place preference paradigm. Fudala et al. (1985) reported that in certain individual rats, nicotine produced a conditioned place aversion, but this observation was not confirmed in a subsequent study (Fudala and Iwamoto 1985). In addition, as Stolerman and D'Mello (1981) have observed, the ability of a substance to produce a CTA does not predict its aversive properties in other behavioural tests. For example, although lithium chloride injection produces both CTAs and conditioned place aversions, certain drugs (e.g. morphine and $d$-amphetamine) produce CTAs but are nevertheless self-administered and produce CPPs (Sherman et al. 1980; Stolerman and D'Mello 1981; Mucha and Iversen 1984). Finally, monkeys and dogs will sometimes selfadminister emetic doses of nicotine (Deneau and Inoki 1967; Risner and Goldberg 1983).

In man, tolerance develops to the dysphoric effects of nicotine (Beckett et al. 1971), and this may be an important determinant of chronic tobacco use. In rats, the acute behavioural actions of nicotine are markedly altered by a few 
daily treatments with the drug, and in tolerant animals, a stimulant action predominates (Clarke and Kumar 1983a, b). In experiment 1, nicotine failed to produce a detectable CPP even in rats which had received several injections of the drug prior to testing. In this case, however, the lack of demonstrable reinforcement may reflect the repeated administration of drug prior to the first exposure to the conditioning apparatus.

In conclusion, the failure to observe nicotine-induced conditioned place preference suggests that the phenomenon is not robust. Possibly, it is limited to "biased" shuttle boxes, in which shifts of side preference may occur independently of drug-induced reinforcement.

Acknowledgements. P.B.S. Clarke is an MRC Postdoctoral Fellow. Supported by the Medical Research Council of Canada (MT-4674).

\section{References}

Beckett AH, Gorrod JW, Jenner P (1971) The effect of smoking on nicotine metabolism in vivo in man. $J$ Pharm Pharmacol 23:62S-67S

Bozarth MA, Wise RA (1981) Heroin reward is dependent on a dopaminergic substrate. Life Sci $29: 1881-1886$

Clarke PBS (1984) Chronic central nicotinic blockade after a single injection of the bisquaternary ganglion blocking drug chlorisondamine. Br J Pharmacol 83:527-535

Clarke PBS, Kumar R (1983a) The effects of nicotine on locomotor activity in non-tolerant and tolerant rats. $\mathrm{Br} \mathbf{J}$ Pharmacol 78:329-337

Clarke PBS, Kumar R (1983b) Characterisation of the locomotor stimulant action of nicotine in tolerant rats. $\mathrm{Br} \mathrm{J}$ Pharmacol $80: 587-594$

Clarke PBS, Kumar R (1984) Some effects of nicotine on food and water intake in undeprived rats. $\mathrm{Br} \mathrm{J}$ Pharmacol $82: 233-239$

Cox BM, Goldstein A, Nelson WT (1984) Nicotine self-administration in rats. Br J Pharmacol 83:49-55

Deneau GA, Inoki R (1967) Nicotine self-administration in monkeys. Ann NY Acad Sci 142:277-279

Dunnett CW (1955) A multiple comparison procedure for comparing several treatments with a control. J Am Stat Assoc $50: 1096-1121$

Fudala PJ, Iwamoto ET (1985) Nicotine-induced conditioned place preference (CPP): dose-response, temporal requirements and lack of tolerance development. Soc Neurosci Abstr 11(2):378.8

Fudala PJ, Teoh KW, Iwamoto ET (1985) Pharmacologic characterization of nicotine-induced conditioned place preference. Pharmacol Biochem Behav 22:237-241
Goldberg SR, Spealman RD, Goldberg DM (1981) Persistent high rate behavior maintained by intravenous self-administration of nicotine. Science 214(4520):573-575

Henningfield JE, Goldberg SR (1983) Control of behavior by intravenous nicotine injections in human subjects. Pharmacol Biochem Behav 19:1021-1026

Kirk RE (1968) Experimental design: Procedures for the behavioral sciences. Brooks/Cole, Belmont, CA, USA

Kumar R (1972) Morphine dependence in rats: secondary reinforcement from environmental stimuli. Psychopharmacology 25:332-338

Kumar R, Pratt JA, Stolerman IP (1983) Characteristics of conditioned taste aversion produced by nicotine in rats. Br J Pharmacol 79:245-253

Laffan RJ, Borison HL (1957) Emetic action of nicotine and lobeline. J Pharmacol Exp Ther 121:468-476

Mithani S, Martin-Iverson MT, Phillips AG, Fibiger HC (1986) The effects of haloperidol on amphetamine- and methylphenidate-induced conditioned place preferences and locomotor activity. Psychopharmacology 90:247-252

Mucha RF, Iversen SD (1984) Reinforcing properties of morphine and naloxone revealed by conditioned place preferences: a procedural examination. Psychopharmacology $82: 241-247$

Phillips AG, LePiane FG (1980) Reinforcing effects of morphine microinjection into the ventral tegmental area. Pharmacol Biochem Behav 12:965-968

Risner ME, Goldberg SR (1983) A comparison of nicotine and cocaine self-administration in the dog: fixed-ratio and progressive-ratio schedules of intravenous drug infusion. J Pharmacol Exp Ther 224:319-326

Sherman JE, Roberts T, Roskam SE, Holman EW (1980) Temporal properties of the rewarding and aversive effects of amphetamine in rats. Pharmacol Biochem Behav 13:597-599

SPSSX User's Guide, SPSS Inc., Chicago, IL

Spyraki C, Fibiger HC, Phillips AG (1982a) Cocaine-induced place preference conditioning: lack of effects of neuroleptics and 6hydroxydopamine lesions. Brain Res 253:195-203

Spyraki C, Fibiger HC, Phillips AG (1982b) Dopaminergic substrates of amphetamine-induced place preference conditioning. Brain Res 253:185-193

Stolerman IP (1986) Psychopharmacology of nicotine: stimulus effects and receptor mechanisms. In: Iversen LL, Iversen SD, Snyder SH (eds) Handbook of psychopharmacology, vol 19. Plenum, New York (in press)

Stolerman IP, D'Mello DG (1981) Oral self-administration and the relevance of conditioned taste aversions. In: Thompson $\mathrm{T}$, Dews PB, McKim WA (eds) Advances in behavioural pharmacology, vol 3. Academic, New York, pp 169-214

Received April 8, 1986 / Final version November 10, 1986 